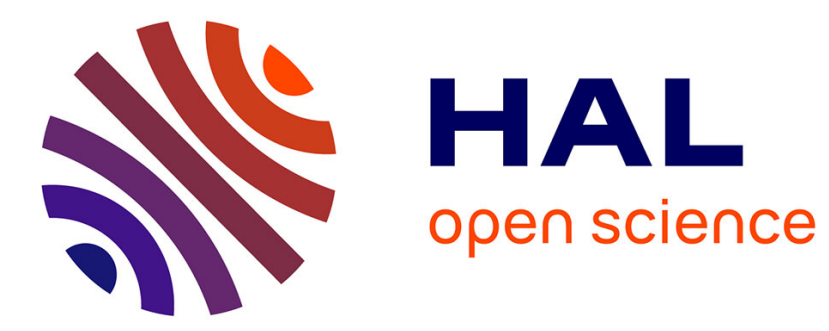

\title{
New schemes of dynamic preservation of diversity. Remarks on stability and topology
}

\author{
Evariste Sanchez-Palencia, Jean-Pierre Françoise
}

\section{To cite this version:}

Evariste Sanchez-Palencia, Jean-Pierre Françoise. New schemes of dynamic preservation of diversity. Remarks on stability and topology. Acta Biotheoretica, 2018, pp.1-13. 10.1007/s10441-019-09353-z . hal-02289362

\section{HAL Id: hal-02289362 https://hal.sorbonne-universite.fr/hal-02289362}

Submitted on 16 Sep 2019

HAL is a multi-disciplinary open access archive for the deposit and dissemination of scientific research documents, whether they are published or not. The documents may come from teaching and research institutions in France or abroad, or from public or private research centers.
L'archive ouverte pluridisciplinaire HAL, est destinée au dépôt et à la diffusion de documents scientifiques de niveau recherche, publiés ou non, émanant des établissements d'enseignement et de recherche français ou étrangers, des laboratoires publics ou privés. 


\title{
New schemes of dynamic preservation of diversity. Remarks on stability and topology.
}

\section{Evariste Sanchez-Palencia ${ }^{1}$}

Jean-Pierre Françoise ${ }^{2}$

\begin{abstract}
-
We address the biological dynamics problem of the persistence of several species in conditions of non-existence of an equilibrium, including an example of stabilization by predation and the very controversial "competitive exclusion", (which depends on the precise definition of persistence). We give normal forms for various examples of such (essentially dynamical) persistence and comments on the involved topology, which implies the presence of exceptional heteroclinic connections binding equilibria on the boundary.
\end{abstract}

Keywords: stability, biological dynamics, persistence,

\section{1-Introduction}

Persistence is obviously the capacity for several species to coexist in some habitat in a sustainable way. In the framework of biological dynamics, there are several mathematical definitions of it (and even several related concepts, as persistence and preservation), but, roughly speaking, persistence amounts to existence of an attractor with non-zero populations of each one of the species; precisions and references are given later (section 4, General comment). In usual examples, there is an interior equilibrium point (i. e. with non-zero populations); if this equilibrium is stable, it is the attractor itself, otherwise it is disjoint of the

\footnotetext{
${ }^{1}$ Sorbonne Université, UPMC Univ Paris 06 and CNRS, UMR 7190, Institut Jean Le Rond d'Alembert, F-75005, France, sanchez@dalembert.upmc.fr

${ }^{2}$ Sorbonne Université, CNRS, Université de Paris, Laboratoire Jacques-Louis Lions (LLL), F-75005 Paris, France Jean-Pierre.Francoise@upmc.fr
} 
attractor; but the presence of an equilibrium is even necessary accordingly to certain strict definitions of persistence, for topological reasons. Other less strict definitions are compatible with attractors without interior equilibrium point. Historically, these new definitions (McGehee and Armstrong 1977) were developed in relation with the very controverted "exclusion principle" (references are given later, in section 3), according to which two different predators cannot live on the same prey, which is also addressed hereafter (section 3). In recent times, several explicit examples of persistence without internal equilibrium were found (Lherminier and Sanchez-Palencia 2015, Sanchez-Palencia and Françoise 2019) revisiting these topics in an explicit computational way.

This communication (initially conceived as an oral presentation to the SFBT colloquium 2018) constitutes both an abstract of Sanchez-Palencia and Françoise 2019 and a prolongation of it. The new contributions are concerned with the "normal forms" (i. e. elementary models exhibiting the main topological features) in sections 2 and 3 and the pedagogical exercise given in "section 5complement". But the "general comment" of section 4 is probably most interesting (although somewhat analogous to those in the previous publication).

This communication is (although computational) mainly qualitative and topological, exhibiting (for reasons which will be explicit in the "general comment" of section 4) systems with (at least) three species not having internal equilibrium i. e., such that there does not exist a point (with non-zero populations of each one of the species), giving an equilibrium (either stable or not). Consequently, the attractor is not a point, but necessarily a more complex structure, (the variety of possibilities is very large, including strange attractors, but the examples given in this paper only involve stable cycles, which are the simplest of them). See for instance, Hirsch Morris, Smale, Stefen, Devaney, Robert L. (2013). The examples and patterns are mainly issued on computations with very precise values of the various parameters (then allowing, in particular, verification). Obviously, these structures change with the parameters, but are stable at least by small changes of their values (classical structural stability of cycles) so that they enjoy some generality.

The plan of the communication is as follows. Two numerical examples are given in sections 2 and 3, concerning models of Stabilization of populations by predation and two predators and one prey, respectively. It follows a "General 
comment" (section 4) including references and a complement to exhibit the role of topology (section 5).

Our basic model of predation for one prey $\mathrm{x}$ and one predator $\mathrm{z}$ is a variant of the Rosenzweig - MacArthur model:

$$
\left\{\begin{array}{c}
x^{\prime}=b x\left(1-\frac{x}{p}\right)-z d \operatorname{Tanh}(a x / d) \\
z^{\prime}=-c z+d z \operatorname{Tanh}(a x / d)
\end{array}\right.
$$

which may be understood as follows. In the absence of the predator $z$, the population of the preys $\mathrm{x}$ is submitted to a "logistic equation" with natural growth $b$ and equilibrium population (with its own resources) $p$. In the absence of preys, the population of predators $z$ decays at the ratio $c$. The term of interaction $\mathrm{zdTanh}(\mathrm{ax} / \mathrm{d})$ accounts for the quantity of preys consumed per unit of time. Each predator eats a quantity of preys which is proportional to the number of preys (the coefficient of proportionality "a" is the vulnerability of the preys, or the efficacy of the process) when the number of preys is small. But when the preys are abundant, this number remains lower than an upper bound $d$ (the satiety of the predator). Indeed, the function $d \operatorname{Tanh}(a x / d)$ describes a transition between ax for small $x$ and $d$ for large $x$. The main (unessential) difference with more usual models is the use of the function tanh instead of an algebraic fraction; in fact tanh approaches better the basic pattern of a linear function truncated by a satiety threshold because of the exponential convergence to the asymptote (visual evidence of this fact may be seen in fig 1 of Sanchez-Palencia and Lherminier 2019). Other explanations and comments on the mathematical models (including cases with several preys) may be seen in the Appendix (section 6) of Sanchez-Palencia and Françoise 2019.

\section{2.- Stabilization of populations by predation.}

We consider an example inspired by the (purely descriptive) paper [Paine 1966] involving stabilization of (unstable) diversity by predation. Here is a citation: "Local species diversity is directly related to the efficiency to which predators prevent the monopolization of the major environmental requisites by one species".

The model for two preys $x, y$ and a predator $z$ is somewhat analogous to (1). Each predator (each individual) eats a quantity of each species of preys which is proportional to the number of its individuals (the coefficients of proportionality are a and $b$, which may be described as the respective vulnerabilities) when the number of preys 
is small. But when the preys are abundant, this total quantity remains lower than an upper bound $d$ (the satiety of the predator, which depends on the total quantity of preys available, not of each one separately). This gives a term of the form $d \operatorname{Tanh}((a x+b$ $y) / d$ ). This function describes a transition between $a x+b$ for small values of $a x+b y$ and $d$ for large $a x+b y$. This total quantity of eaten preys is clearly shared between the two preys proportionally to the populations pondered by the corresponding vulnerabilities. Otherwise, in the absence of predators, the terms of natural growth up to the population $p$ of equilibrium with the subsistence (logistic terms) take the form $e$ $x(1-(x+Y y) / p)$ and $f y(1-(y+\delta x)=p)$ where $Y$ and $\delta$ denote coefficients of interaction between the two preys concerning their resources. Completely independent preys, rabbits ant truits for instance, amounts to $Y=\delta=0$, whereas two races of rabbits corresponds to $Y=\delta=1$. Here e and $f$ are the natural growth rates, taken equal to 1 in the sequel.

Specifically, we consider the system

$$
\left\{\begin{array}{c}
\mathrm{x}^{\prime}=\mathrm{x}[1-((\mathrm{x}+\Upsilon \mathrm{y}) / \mathrm{p})]-\mathrm{z} \operatorname{Tanh}(\mathrm{ax}+\mathrm{by}) \frac{\mathrm{ax}}{\mathrm{ax}+\mathrm{b} y} \\
\mathrm{y}^{\prime}=\mathrm{y}[1-((\mathrm{y}+\delta \mathrm{x}) / \mathrm{q})]-\mathrm{z} \operatorname{Tanh}(\mathrm{a} \mathrm{x}+\mathrm{b} y) \frac{\mathrm{b} y}{\mathrm{ax}+\mathrm{by}} \\
z^{\prime}=z[-c+\operatorname{Tanh}(a x+b y)]
\end{array}\right.
$$

with the values of the parameters: $a=1.2, b=1.0, c=0.6, d=1.0, p=5.0, q=$ $4.0, \gamma=0.6, \delta=0.9$. These values show that $x$ are advantaged with respect to the $y$ because they have a larger equilibrium population with their subsistence and a smaller influence of the other (but the specific values may be changed in some extent). When the predator $z$ is not present, this obviously leads to the disappearing of the $y$ (there is a point attractor on the $x$ axis). We then add a predator $z$ of both $x$ and $y$, to which the $x$ are more vulnerable than the $y$. It then appears, (for certain ranges of the parameters) a three dimensional attractor (persistence of $x, y$ and $z$ ). There is no equilibrium point with three non-vanishing populations, and it appears a heteroclinic orbit binding (for $t$ tending to $+\infty$ and $-\infty$ ) two (unstable) equilibrium points on the planes $x, z$ and $y, z$. 


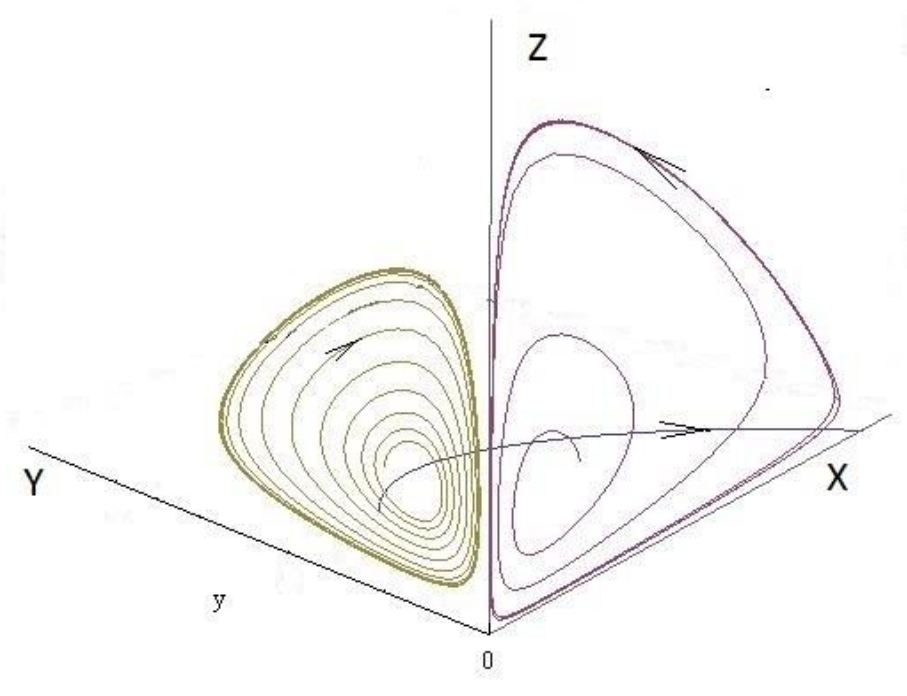

Fig 1-Stabilisation via predation. Examples of orbits inside the planes $x, y$ (no persistence) $x, z$ et $y, z$ (persistence).

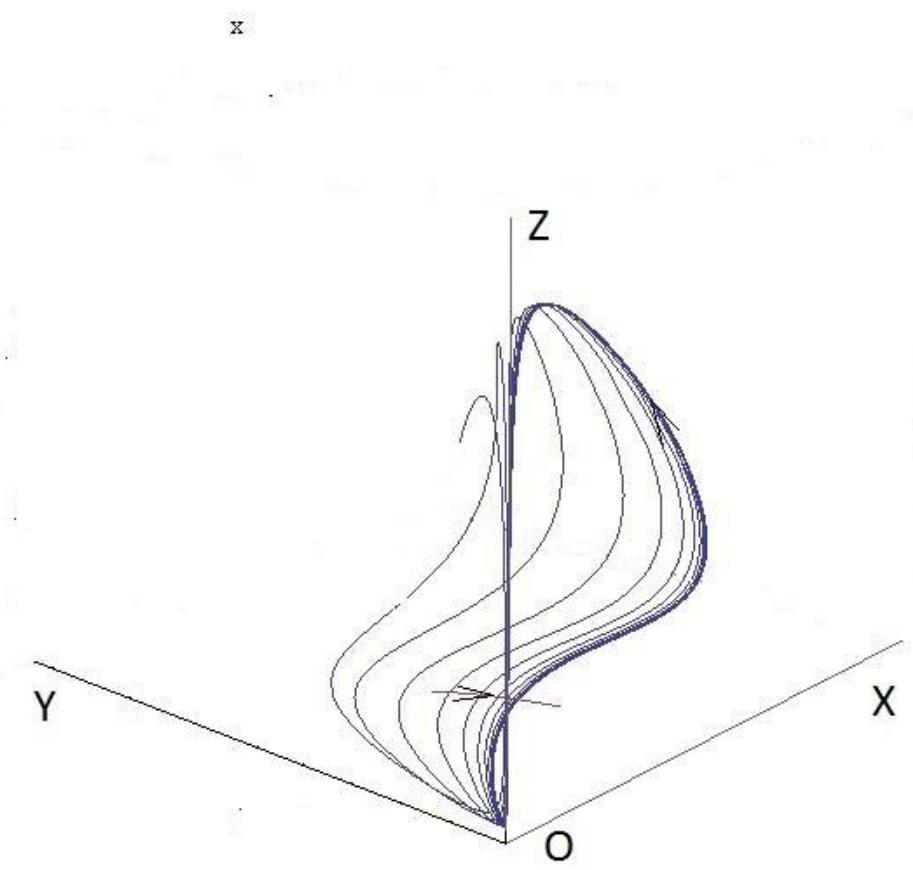

Fig 2-Stabilization by predation. The picture exhibits an orbit tending towards an attractor (stable cycle, persistence of $x, y, z$ ) and a heteroclinic orbit binding the unstable equilibrium points on the planes $x, z$ and $y, z$ for $t$ tending to $+\infty$ and $-\infty$. 
The topological structure of the phase portrait is easily understood with the next " normal form ", i. e. a simplified model obtained by distorting the domain, which becomes in cylindrical coordinates the slice $-1<Z<1$. The planes $(x, z)$ and $(y, z)$ become the vertical planes $Z=-1$ et $Z=1$; in addition, there is a rotation with constant angular speed $\theta^{\prime}=1$. The phase portrait in the meridian plane $(Z, r)$ is:

$$
\left\{\begin{array}{c}
r^{\prime}=r-r^{3} \\
Z^{\prime}=\left(1-Z^{2}\right)\left(r^{2}-Z-1\right)
\end{array}\right.
$$

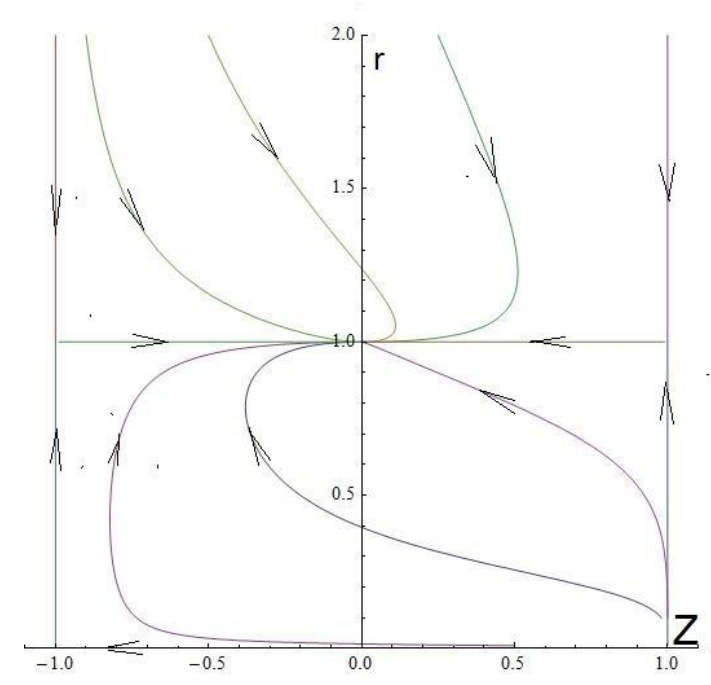

Fig 3- Stabilization by predation. Normal form. Phase portrait in the meridian plane $(r, \vartheta)$ (one must also imagine a rotation with constant angular speed around axis $Z$, which is also a heteroclinic orbit). The point $(0,1)$ is the cyclic attractor). There is no equilibrium point with positive populations.

This system, augmented with $\theta^{\prime}=1$, can be derived from the following system (4) via a change from cartesian $(X, Y, Z)$ to cylindrical $(r, \theta, Z)$ coordinates:

$$
\left\{\begin{array}{c}
X^{\prime}=-Y+X\left(1-X^{2}-Y^{2}\right) \\
Y^{\prime}=X+Y\left(1-X^{2}-Y^{2}\right) \\
Z^{\prime}=\left(1-Z^{2}\right)\left(X^{2}+Y^{2}-Z-1\right)
\end{array}\right.
$$

This obviously accounts for system (3) on the meridian plane and a rotation with constant angular speed.

It is apparent that there is no internal equilibrium point and that the segment $r=0$ is a heteroclinic orbit.

Moreover, changing a little the values of the parameters $(\gamma=0.7$ instead of $\gamma$ $=0.6$ ), it appears a bi-stability phenomenon: there is a new (unstable !) cycle; its stable manifold is the boundary between two attraction basins, the previous 
(slightly modified) one and a new one with sufficiently small $x / z$, with attractor on the $(y, z)$ plane.

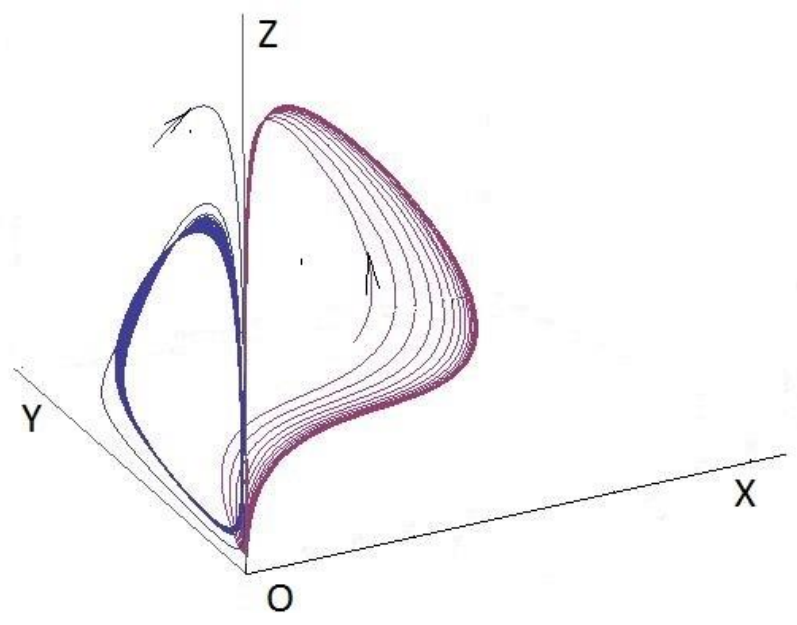

Fig 4-Stabilization by predation. Bi-stability case. Orbits are asymptotic either to an attractor with three non-zero populations or to another one with $x=0$. Persistence is conditional. There is again a heteroclinic orbit and no equilibrium point with three non-zero populations.

The corresponding normal form and phase portrait in the meridian plane $(r, Z)$ of the cylindrical coordinates $(r, \theta, z)$ are analogous to the previous ones, with the system

$$
\left\{\begin{array}{c}
r^{\prime}=r-r^{3} \\
Z^{\prime}=1.5\left(1-Z^{2}\right)\left(1-0.5 r^{2}+Z\right)
\end{array}\right.
$$

Instead of (3) and Fig 5 instead of Fig 3: 


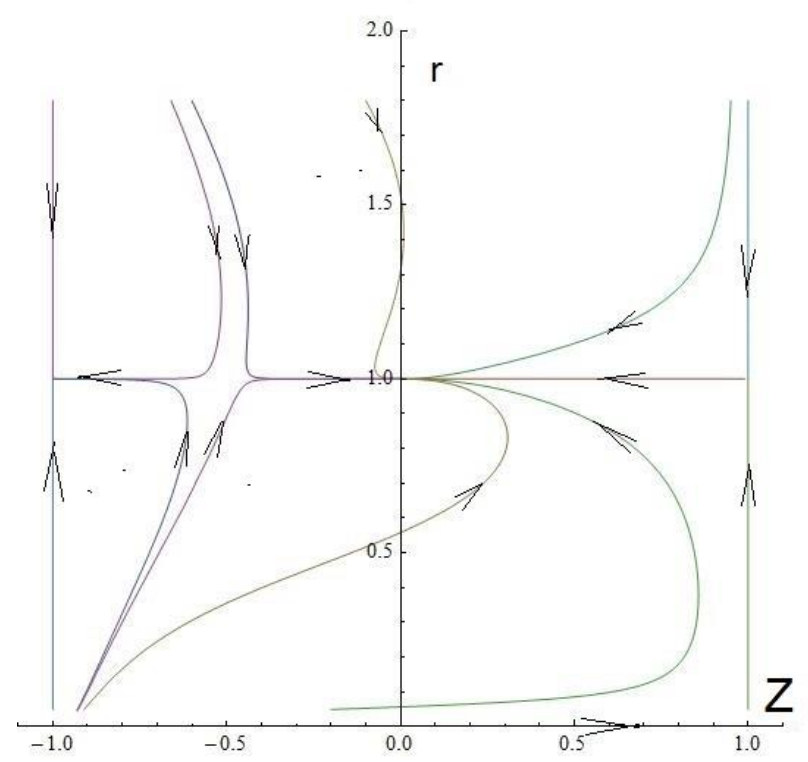

Fig 5- Stabilization by predation. Normal form in the bi-stability case. The two attractors are two circles in cylindrical $(r, \vartheta, Z)$ coordinates; their intersections with the meridian plane are the points $(0,1)$ et $(-1,1)$. There is also a an unstable cycle $(-0.5,1)$; its stable manifold is the interface between the two attraction basins. There is again a heteroclinic orbit $r=0$ and no equilibrium point with positive populations.

An explanation of this phase portrait is the following. As the vulnerabilities are somewhat large, the general context involve cycles (instead of equilibrium points). Starting from a large population of predators $z$ and scarce preys, $z$ obviously decreases; as the $x$ preys are more vulnerable, the $y$ ones are the first to take off, so that the orbit takes off from the vicinity of the $z$ axis towards the $y$ axis. When the populations of preys are sufficiently large, the significant term is their nonlinear interaction, which turns out into an advantage of the $x$, (so that the orbit curves towards the $x$ axis). Next, for sufficiently large $x$ and $y$, the population of preys starts growing; there is a frenetic rise of $z$, which exhausts the preys (we are at the apex of the orbit) and this entails the fall of $z$, closing the cycle. The bi-stability is also easily understood: indeed, provided that the initial position of the populations $y$ and $z$ are sufficiently large, the scarce $x$ is not able to impose their advantage to the $y$, and the attractor only involves $y$ and $z$.

\section{3-Two predators and one prey. Dynamical persistence.}

Let us comment a little again on the very controversial case of two predators and one prey, which leads, in the framework of static attractors, to the so-called 
"competitive exclusion principle", whereas it is false in the larger framework of cyclic attractors (with a heteroclinic orbit not leading to the attractor). As a matter of fact, coexistence of both predators is possible in the case of cycles, provided there are advantages and disadvantages which balance along a (stable) cycle ([McGehee and Armstrong 1977], [Armstrong and McGehee 1980, [Lherminier and Sanchez-Palencia 2.015], [Sanchez-Palencia and Françoise 2019]).

Specifically, we consider the system for a prey $\mathrm{x}$ and the two predators $z_{1}$ and $z_{2}$ :

$$
\left\{\begin{array}{c}
x^{\prime}=x\left(1-\frac{x}{p}\right)-z_{1} d_{1} \operatorname{Tanh}\left(x / d_{1}\right)-z_{2} d_{2} \operatorname{Tanh}\left(x / d_{2}\right) \\
z_{1}^{\prime}=-c_{1} z_{1}+z_{1} d_{1} \operatorname{Tanh}\left(x / d_{1}\right) \\
z_{2}^{\prime}=-c_{2} z_{2}+z_{2} d_{2} \operatorname{Tanh}\left(x / d_{2}\right)
\end{array}\right.
$$

with the values of the parameters:

$d_{1}=1, d_{2}=2, p=4, c_{1}=0.55, c_{2}=0.7$.

It is apparent that $z_{1}$ has a larger satiety bound than $z_{2}$, as well as a larger natural mortality. They are an advantage and a drawback of different nature, allowing a balance along a cycle.

The phase portraits on the coordinate planes are depicted in Fig 6 . The planes $x z_{1}$ and $x z_{2}$ correspond to the presence of only one of the predators. There is a stable three-dimensional cycle which is a global attractor (the attraction basin is the whole strictly positive cone), shown in Fig 7. 


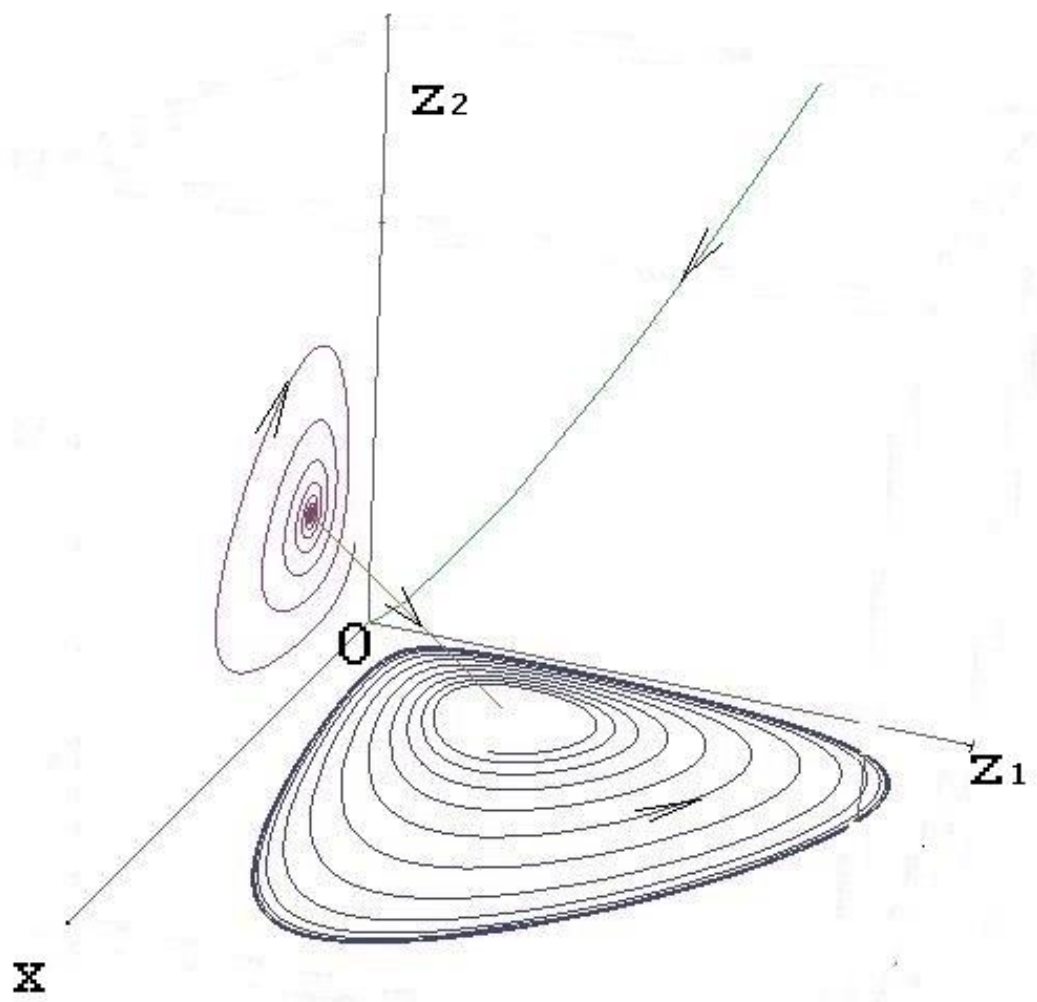

Fig 6-Two predators $z_{1}$ and $z_{2}$ and one prey $x$. Phase portrait of the dynamics on the coordinate planes. On $x, z_{1}$ there is a cyclic attractor (along with an unstable equilibrium), on $x, z_{2}$ there is a point attractor, and on $z_{1}, z_{2}$ the attractor is the origin. These attractors are all transversally unstable (sending to the global attractor with three non-zero populations of Fig 7). Here we also plotted a heteroclinic orbit binding the equilibria in the planes $x, z_{1}$ and $x, z_{2}$.

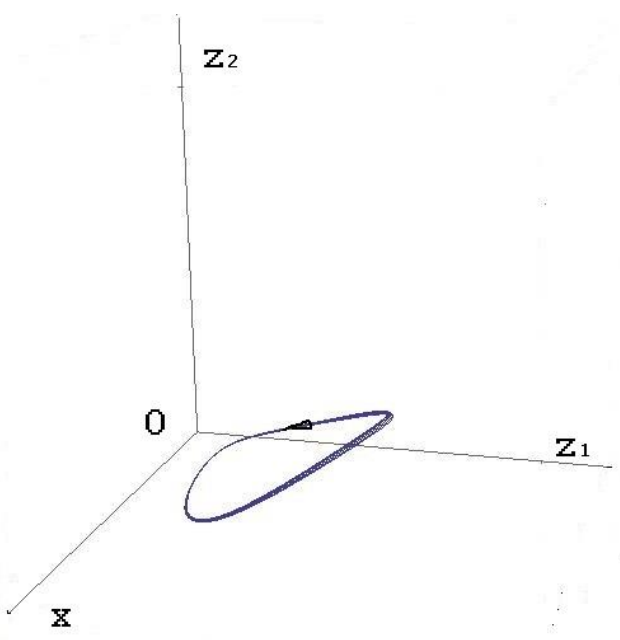


Fig 7- Two predators $z_{1}$ and $z_{2}$ and one prey $x$. The global attractor with three non-zero populations.

In the present example, the orbits converge towards the attractor spiraling as is shown in the artist view of Fig 8 . Note that this scheme is consistent with Figs. 6 and 7.

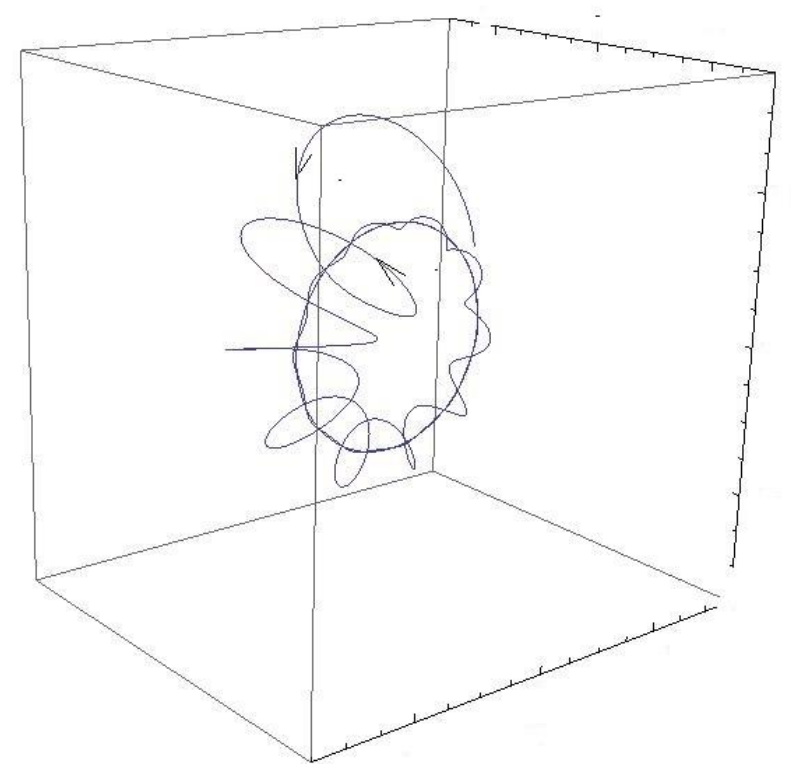

Fig 8-Two predators $z_{1}$ and $z_{2}$ and one prey $x$. Artist view of the convergence of orbits to the attractor.

The normal form and the phase portrait in the meridian plane of the cylindrical coordinates (analogous to (a) and (b)) are:

$$
\left\{\begin{array}{c}
r^{\prime}=Z r-r^{3} \\
Z^{\prime}=3\left(0.2-r^{2}\right)\left(1-Z^{2}\right)
\end{array}\right.
$$

This system, augmented with $\theta^{\prime}=1$, can be derived from the following system (8) via a change from cartesian $(X, Y, Z)$ to cylindrical $(r, \theta, Z)$ coordinates:

(8) $\left\{\begin{array}{c}X^{\prime}=-Y+X\left(Z-X^{2}-Y^{2}\right) \\ Y^{\prime}=X+Y\left(1-X^{2}-Y^{2}\right) \\ Z^{\prime}=3\left(0.2-X^{2}-Y^{2}\right)\left(1-Z^{2}\right)\end{array}\right.$ 
This obviously accounts for system (7) on the meridian plane and a rotation with constant angular speed.

The phase portrait in the meridian plane is depicted in Fig 9. It is clear that the convergence towards the limit cycle (the attracting point in the meridian plane) is made by spiraling around it (combination of the scheme depicted in the figure and the uniform rotation $\theta^{\prime}=1$ ).

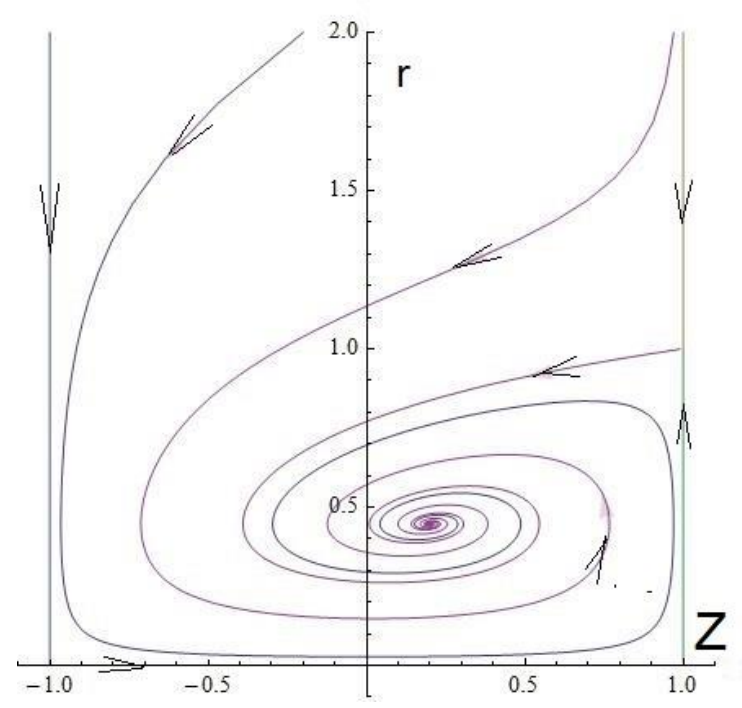

Fig 9-Two predators $z_{1}$ and $z_{2}$ and one prey $x$. Phase portrait of the normal form (c) in the plane $z$, $r$. One must also imagine a rotation with constant angular speed around axis $Z$, which is also a heteroclinic orbit

It should be noted, as before, that there is no internal equilibrium and that the segment $(-1,1)$ of the $Z$ axis is a heteroclinic orbit.

\section{4.- General comment}

It is obvious that the heteroclinic orbit in the previous examples, as it binds (for $t$ tending to $-\infty$ and $+\infty$ ) two points on the outer boundary, is not in the attraction basin of the global attractor. It is constituted by "forbidden points" (as well as all points on the boundary - there is no spontaneous generation). This does not entail any practical difficulty, as only a set with zero measure is involved. As a matter of fact, the very mention of the heteroclinic orbit is a curiosity which should be omitted. 
The strict definition of preservation (see for instance Hofbauer J. and Sigmund K. 1988, chap. 13) implies the convergence towards the attractor of orbits issued from any point of the positive cone, which (after some technical work avoiding neighborhoods of infinity and of the boundary) is homeomorphic to a ball. It follows (from a classical topological theorem) that the vector field must vanish at one interior point at least. In other words, it is widely known and explicitly stated (see Hofbauer J. and Sigmund K. 1988, theorem 13.3.1, p 158) that (strict) persistence implies the existence of an (stable or not) internal equilibrium. It follows that a vector field without internal equilibrium cannot be (strictly) preserving. It is easily seen that the system with two predators and one prey has no internal equilibrium (the corresponding algebraic system has two equations with one unknown), and this constitutes one of the proofs of the competitive exclusion principle (there are other proofs, such as in Brauner Castillo-Chavez 2012, sect 5.11, p 217), using the transversal stability of one of the equilibria on the boundaries, which, as we saw, does not exclude the possibility of a cyclic attractor).

Since 1977 (before the computer revolution). McGehee et Armstrong noticed that the attraction basins of cyclic attractors are not homeomorphic to balls, they are manifolds with boundary with Euler characteristic equal to 0 (roughly speaking, they are homeomorphic to a solid torus). They also gave a (somewhat complex and not much explicit) proof of the existence of a preserving system of two predators and one prey using a larger (not strict) definition of persistence (existence of an internal attractor). This work, which was in the past widely cited to support some skepticism concerning the competitive exclusion principle, is nowadays somewhat forgotten and is not quoted in recent literature.

Since 2015, using ideas of Ph. Lherminier on a plausible ecologic mechanism, explicit examples of non-exclusion were given, followed by other examples of (not strict) preservation without internal equilibrium (see Lherminier and Sanchez-Palencia 2015 and Sanchez-Palencia and Françoise 2019). Otherwise, the definition of McGehee and Armstrong of preservation (existence of an internal attractor) is no more satisfactory, as other attractors may be present on the boundary.

In our opinion, the definition of a satisfactory and useful idea of preservation is widely open, as such a definition should account for several new examples. 


\section{5- Complement. An exercise exhibiting the role of topology}

In order to understand the topological constraints and their implication in that kind of problems, we consider the following problem, which is essentially equivalent to a simplified form of the above ones, after some handling.

In the plane $\mathrm{x}, \mathrm{y}$ there is a dynamical system depending on a parameter $\lambda \in$ $[0,1]$ with a global attractor $C(\lambda)$. We first consider the case when, for any $\lambda \in$ $[0,1], C(\lambda)$ is a stable cycle. According to the index theory in the plane, there is at least an interior equilibrium $x_{0}(\lambda), y_{0}(\lambda)$ (See Fig 10).

Fig 10

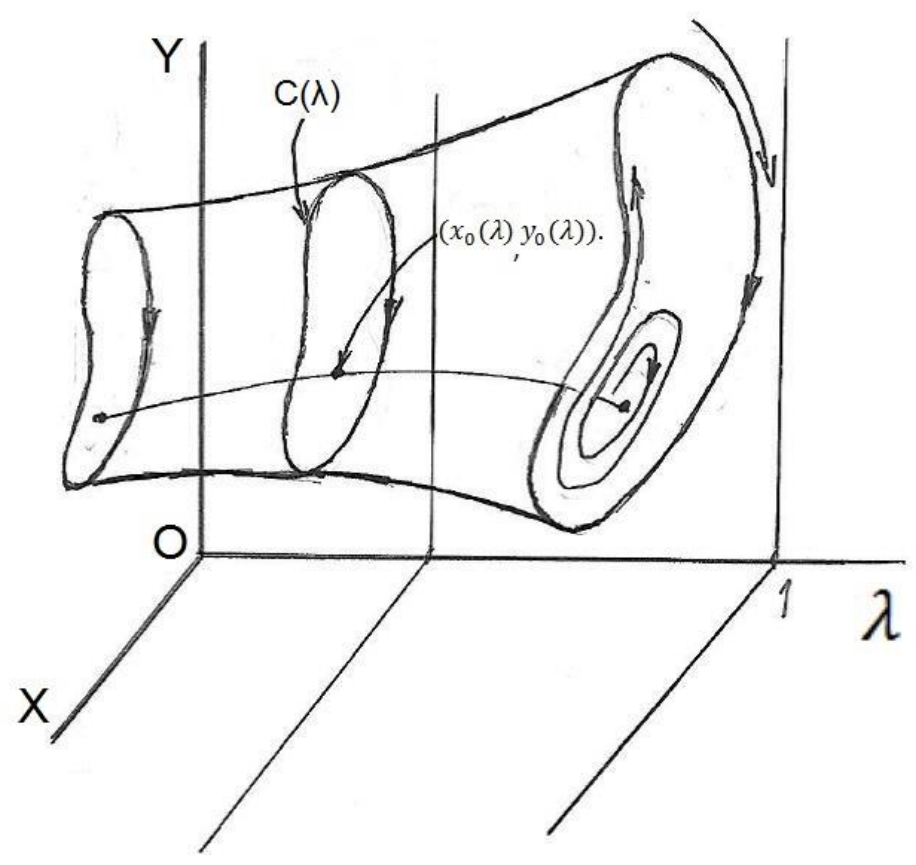

The problem is to transform the parameter $\lambda$ in a new dynamic variable in order to obtain a dynamical system in $x, y, \lambda$, with invariant planes $\lambda=0$ and $\lambda=1$ and a global attractor, without internal equilibrium. We then search for a new equation of the form (in order to keep invariant $\lambda=0$ and $\lambda=1$ ):

$$
\lambda^{\prime}=f(x, y, \lambda)=\lambda(1-\lambda) \mathrm{F}(x, y, \lambda),
$$

Moreover, we impose that the cycles in $\lambda=0$ and $\lambda=1$ be transversally unstable (in order the global attractor not to be on the boundaries $\lambda=0$ and $\lambda=1$ ). It is easily seen (variation equations) that this amounts to: 


$$
\text { (10) }\left\{\begin{array}{l}
\int_{C(0)} F(x, y, 0) d s>0 \\
\int_{C(1)} F(x, y, 1) d s<0
\end{array}\right.
$$

The constraint of non-existence of internal equilibrium amounts to:

(11) $F\left(x_{0}(\lambda), y_{0}(\lambda), \lambda\right) \neq 0 \quad$ for $\lambda \in[0,1]$.

We see that there is a large choice of smooth functions F satisfying (10) and (11). Moreover, In the particular (but general, by means of a diffeomorphism) case when $x_{0}(\lambda), y_{0}(\lambda)$ is independent of $\lambda$, the line of this two-dimensional equilibria is a segment parallel to the $\lambda$ axis, and it is a heteroclinic orbit of the three-dimensional dynamics, so allowing the presence of a three-dimensional attractor without internal equilibrium.

Let us now consider the same problem in the case when for any $\lambda \in[0,1]$, the attractor $C(\lambda)$ is the equilibrium $x_{0}(\lambda), y_{0}(\lambda)$ itself. In that case, the conditions (10) becomes

$$
\left\{\begin{array}{l}
F\left(x_{0}(0), y_{0}(0), 0\right)>0 \\
F\left(x_{0}(1), y_{0}(1), 1\right)<0
\end{array}\right.
$$

which is incompatible with (11) for the classical (topological!) property that a continuous function on an interval cannot take values with opposite signs without vanishing in an intermediate point.

This shows the drastic difference between the cyclic and point frameworks concerning the topological possibilities.

\section{References}

Armstrong R. A. and McGehee R (1980) Competitive exclusion. The American Naturalist 115, p 151-170.

Brauner F. and Castillo-Chavez C. (2012) Mathematical models in population biology and epidemiology, Springer. 
Hirsch Morris, Smale, Stefen, Devaney, Robert L. (2013) Differential equations, dynamical systems, and an introduction to chaos. Third edition. Elsevier/Academic Press, Amsterdam. $x i v+418 p p$.

Hofbauer J. and Sigmund K. (1988). The theory of Evolution and Dynamical Systems, London Math. Soc. Student Texts 7, Cambridge University Press

Lherminier P. and Sanchez-Palencia E (2015) Some remarks and examples on transient processes and attractors in biological evolution. Electronic Jour Diff Equations Conference 22 p. 63-77.

McGehee R. and Armstrong R. A. (1977) Some mathematical problems concerning the ecological principle of competitive exclusion. Jour. Diff. Eq. 23, p 30-53.

Paine, R. T. (1966) Complexity and species diversity. The American Naturalist 100 ( $\left.N^{\circ} 910\right) p$ 65-75.

Sanchez-Palencia E. and Françoise J. P. (2019) Topological remarks and new examples of persistence of diversity in biological dynamics. Disc. and Cont. Dyn. Syst. Series $S, 12\left(N^{\circ} 6\right)$, p1775-1789.

Sanchez-Palencia E. and Lherminier Ph. (2019) Paradoxes of vulnerability to predator in biological dynamics and mediate versus immediate causality. Disc. and Cont. Dyn. Syst. Series S, to appear 